\title{
Low refractive index SiOF thin films prepared by reactive magnetron sputtering
}

F.J. Garcia-Garcia, J. Gil-Rostra, A. Terriza, J.C. González, J. Cotrino, F. Frutos ${ }^{a}$, A.R. González-Elipe, F. Yubero*

Instituto de Ciencia de Materiales de Sevilla (CSIC, Univ. Sevilla). Av. Américo Vespucio 49, E-41092, Sevilla, Spain.

${ }^{a}$ Applied Physics Department, E.T.S. Ingeniería Informática, University of Seville, Avd. Reina Mercedes s/n, E-41012 Seville, Spain

${ }^{*}$ Corresponding author

Email: yubero@icmse.csic.es

\section{Keywords:}

SiOF, thin films, magnetron sputtering, optical properties

\begin{abstract}
We have studied low refractive index fluorine doped silica thin films prepared by reactive magnetron sputtering. Two experimental parameters were varied to increase the porosity of the films, the geometry of the deposition process (i.e., the use of glancing angle deposition) and the presence of chemical etching agents (fluorine species) at the plasma discharge during silica film growth. The microstructure, chemistry, optical properties, and porosity of the films have been characterized by scanning electron and atomic force microscopy, Fourier transform infrared spectroscopy, X-ray photoelectron spectroscopy, UV-Vis, and spectroscopic ellipsometry. It is found that either the deposition at glancing angles or the incorporation of $\mathrm{CF}_{\mathrm{x}}$ species in the plasma discharge during film growth produces a decrease in the refractive index of the deposited silica films. The combined effect of the two experimental approaches further enhances the porosity of the silica films. Finally, the films prepared in a glancing geometry exhibit negative uniaxial birefringence.
\end{abstract}




\section{Introduction}

Porous silica based thin films have strong interest in several technological fields as intermetallic dielectrics in microelectronics due to their low permittivity [1] or as low index layers in antireflective coatings intended for optical applications [2,3]. They also have been extensively used as host materials of active species within their porous structure for the development of sensors [4].

Low refractive index silica thin films have traditionally been prepared by sol-gel methods [3,5] carried out by dip, spin, or spray techniques. These deposition processes typically require heat treatments at several hundred degrees to eliminate organic compounds that remain otherwise in the films after processing. Plasma enhance chemical vapor deposition is a cleaner environmentally process that allows to grow porous silica films at room temperature [6].

Other possibility to get porous silica based thin films is to dope them with $\mathrm{F}$ atoms, a process that makes the bond structure more open leading to a decrease in density $[7,8,9,10,11,12,13,14,15]$. This type of films is mostly prepared by plasma enhanced chemical deposition methods [11,13] using fluorine containing gases $\left(\mathrm{CF}_{4}, \mathrm{SiF}_{4}\right)$ in the plasma discharge, although other wet routes have also been used [15].

On the other hand, Glancing angle deposition (GLAD) has also been extensively used to get silica films with porous structures [16]. In this case, the shadowing effects induced by the nucleation centers that appear in the initial growth stages induce the formation of porous columnar structures. This strategy is extensively used in physical vapor deposition processes such as electron beam evaporation. Although magnetron sputtering is less used for this purpose, this methodology has been also used to get porous structures as recently illustrated by the preparation of porous mixed oxide $\mathrm{W}:$ SiOx electrochromic coatings [17].

In this contribution we describe the synthesis of porous fluorine doped silica thin films prepared by reactive magnetron sputtering (MS). We have used $\mathrm{Si}$ as a target and $\mathrm{O}_{2}$, $\mathrm{Ar}$, and $\mathrm{CFx}$ gas mixtures (with $\mathrm{CFx}$ either tetrafluorocarbono, $\mathrm{CF}_{4}$ or octafluorocyclobutane, $\mathrm{C}_{-} \mathrm{C}_{4} \mathrm{~F}_{8}$ ). In particular, we investigate the combined effect of glancing angle deposition, Si-F bond formation within the silica bond structure, and the etching processes induced by $\mathrm{CF}_{x} / \mathrm{O}_{2} / \mathrm{Ar}$ mixtures in the magnetron plasma discharge during porous fluorine doped silica thin film growth. The deposition process described in this paper can easily be implemented in any MS equipment. It might be very interesting for industrial processes related to the preparation of antireflective coatings, or other layered systems where low refractive index or enhanced porous silica based films prepared by MS are required.

\section{Experimental}


Porous fluorine doped silica thin films (SiOF) were prepared by reactive MS using a silicon target of 2 inches diameter as source of $\mathrm{Si}$. The magnetron head (GENCOA) was powered with a pulsed $(80 \mathrm{kHz})$ DC source (MPS15 from Milko Angelov Consulting Ltd) with a power of 100-300 W and a voltage of 400-450 V. Mixtures of $\mathrm{Ar}, \mathrm{O}_{2}$, and $\mathrm{CF}_{\mathrm{X}}$ (either $\mathrm{CF}_{4}$ or $\mathrm{C}_{-} \mathrm{C}_{4} \mathrm{~F}_{8}$ ) as reactive gases were dosed with mass flow controllers. Films were grown at normal and glancing geometries (i.e., $\alpha=0^{\circ}$ and $\alpha=$ $80^{\circ}$ respectively, with $\alpha$ the angle between the normal to magnetron target and the normal to surface of the sample) and several mass flow rates for the different gases. The films were grown with constant mass flow rates of $\mathrm{Ar}, \Phi_{\mathrm{Ar}}$, and $\mathrm{O}_{2}, \Phi_{\mathrm{O} 2}$ of 40 and $5 \mathrm{sccm}$, respectively. Regarding $\mathrm{CF}_{\mathrm{X}}$, mass flow rates $\Phi_{\mathrm{CFx}}$ between 0 and $2 \mathrm{sccm}$ were considered. In the following, we will name the samples prepared with $\alpha=0^{\circ}$ without and with fluorine species in the plasma discharge as SiO2-N and SiOF-N, respectively. Similarly, samples prepared in GLAD geometry without and with fluorine species in the plasma discharge will be labeled as SiO2-GLAD and SiOF-GLAD.

Polished $\mathrm{Si}(100)$ wafers and fused silica plates were used as substrates. The distance between substrates and target was $\sim 10 \mathrm{~cm}$. The base pressure of the system was $3.0 \times 10^{-6}$ mbar. The pressure during deposition was fixed at $5.0 \times 10^{-3}$ mbar. The growth rate was about $10 \mathrm{~nm} / \mathrm{min}$. The incorporation of $\mathrm{CF}_{\mathrm{x}}$ species in the plasma discharge did not vary significantly the deposition rate. The substrate temperature during film growth was always below $80^{\circ} \mathrm{C}$.

The magnetron plasma discharge was characterized by Optical Emission Spectroscopy (OES) with a multichannel fiber optic spectrometer (AvaSpec-3648USB2-RM desktop rack with three channels) with $0.07 \mathrm{~nm}$ wavelength resolution. The light was collected with an optical fiber vacuum feedthrough with a collimating lens pointing towards the maximum intensity of the plasma discharge.

Microstructure was evaluated using a field emission scanning electron microscope (FESEM, Hitachi 5200). Surface morphology by atomic force microscopy (AFM) using a Cervantes microscope with Dulcinea electronics from Nanotec. Bond type structure was analyzed with FT-IR spectroscopy using a JASCO spectrometer. The surface stoichiometry of these samples was obtained from XPS measurements using a VG-ESCALAB 210 spectrometer.

The thin film fluorine content was studied by a combined proton Rutherford Backscattering Spectrometry ( $p$-RBS) and Proton induced gamma ray emission (PIGE) analysis. These characterizations were performed at the $3 \mathrm{MV}$ tandem accelerator of National Center for Accelerators (Seville, Spain). p-RBS and PIGE measurements were performed using protons of 1550 and $2330 \mathrm{keV}$, respectively. More details about this characterization can be found elsewhere [18].

The optical transmittance and the refractive index of the porous films were obtained by UV-Vis spectroscopy and spectroscopic ellipsometry. The interference fringes observed in reflection and transmission UV-Vis spectra were fitted using a Cauchy 
law for the wavelength dispersion of the refractive index. Void fraction was evaluated using the Lorentz-Lorenz relation according to the effective medium theory.

Optical analysis of the films deposited on polished $\mathrm{Si}(100)$ wafers was also carried out in a J.A. Woollam VASE spectroscopic ellipsometer. Values of $\Psi$ and $\Delta$ were obtained over the spectral range of 300 to $900 \mathrm{~nm}$, at $2 \mathrm{~nm}$ resolution. As a consistency check of the data, the incidence angle was varied at three angles of incidence: $65^{\circ}, 70^{\circ}$, and $75^{\circ}$ degrees. Optical anisotropic modeling and parameters fitting were done with the WASE32@ program (J.A. Woollam Co. Inc.). Optical response of thin films prepared at normal geometry was modeled with an isotropic description of the refractive index of the film. On the other hand, the optical characteristics of the films prepared at glancing angle deposition were modeled using an anisotropic uniaxial description of the refractive index, considering in-plane $n_{x}$ and out-of-plane $n_{z}$ contribution to the refractive index of the film. The surface roughness layer was assumed to consist of $50 \%$ film material and $50 \%$ voids. For this analysis, we also considered a Cauchy wavelength dispersion of the refractive index.

\section{Results}

\section{$\underline{\text { Plasma diagnosis }}$}

Figure 1 shows a typical OES spectra measured at the maximum intensity of the plasma discharge (i.e., with the optical fiber facing directly the Si target) for the different deposition conditions used in this work. Plasmas with argon-oxygen mixtures are characterized by numerous intense emission lines produced by the $\mathrm{Ar}$ atoms (excited states from $4 p$ levels) and few weak atomic oxygen lines (c.f., at 777 and $845 \mathrm{~nm}$ ) [19]. Apart from the $\mathrm{O}$ and Ar lines, some weak $\mathrm{OH}$ rotational bands were also observed at $\sim 306 \mathrm{~nm}$. These bands resulted from the water desorbed form the chamber walls. Only minor differences were observed when a fluorocarbon gas (either $\mathrm{CF}_{4}$ or $\mathrm{C}-\mathrm{C}_{4} \mathrm{~F}_{8}$ ) was introduced (up to a mass flow rate of $2 \mathrm{sccm}$ ) in the plasma discharge. In this case, lines corresponding to partial decomposition of the fluorine precursors $\left(\mathrm{F}^{*}, \mathrm{C}-\mathrm{F}_{\mathrm{x}}{ }^{*}\right)$ were not observed. Thus, $\mathrm{CF}_{2}$ radicals, important intermediates in fluorocarbon plasma chemistry usually observed in the $220-300 \mathrm{~nm}$ region [20], have not been detected in our experimental conditions. This might be due to the high dissociation of the fluorocarbon gas near the cathode. In addition, a low electron density in the discharge with energies higher than the excitation threshold of $\mathrm{F}$ atoms $(\sim 14.4 \mathrm{eV})$ can also justify the absence of $\mathrm{F}^{*}$ lines in the discharge. Despite the absence of emission lines characteristic of the fluorine species introduced in the plasma discharge, clear influence in the microstructure, chemistry, and optical properties of these films were observed, as we will show below. 


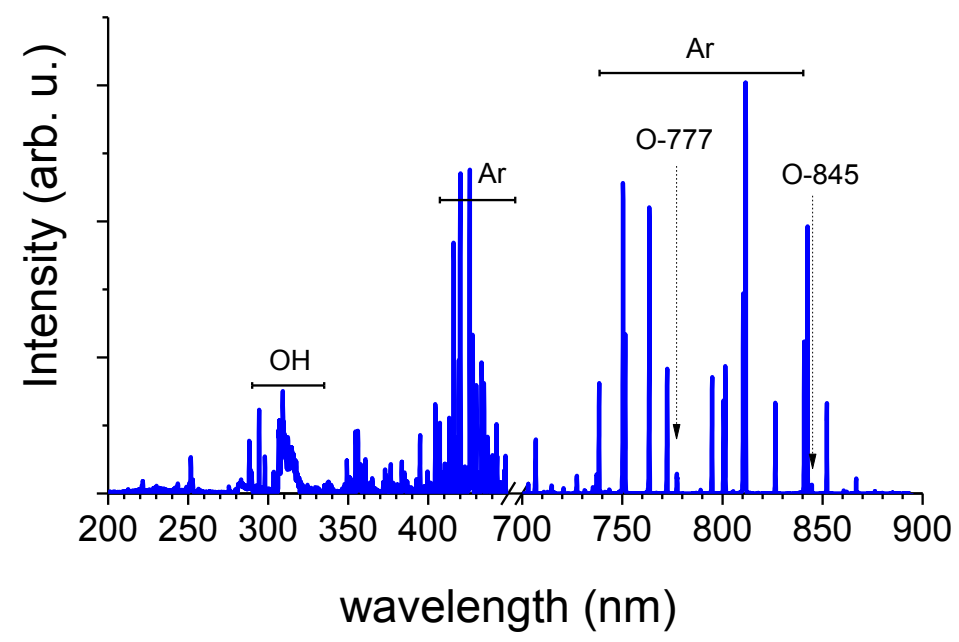

Figure 1. OES spectra measured at the plasma discharge during SiOF film growth by reactive magnetron sputtering.

\section{Microstructure}

Figure 2 (top) shows cross-section SEM micrographs of porous silica based films deposited in normal geometry without (SiO2-N) and with (SiOF-N) fluorine species in the plasma discharge. We observe a columnar growth microstructure typical of magnetron sputtering deposition when fluorine species are not present in the plasma discharge during the film growth. When $\mathrm{CF}_{\mathrm{x}}$ species are incorporated in the discharge (right) a more disordered growth is appreciated with small lateral ramifications between columns. Figure 2 (bottom) shows cross-sectional SEM images of $\mathrm{SiO}_{2}$ samples prepared in glancing geometry without (SiO2-GLAD) and with fluorine species (SiOF-GLAD) in the plasma discharge. In this case, a more open structure than that depicted for the SiO2-N samples was found. However, the incorporation of $\mathrm{CF}_{\mathrm{X}}$ precursor in the plasma discharge does not show a clear effect in the GLAD thin film microstructure. 

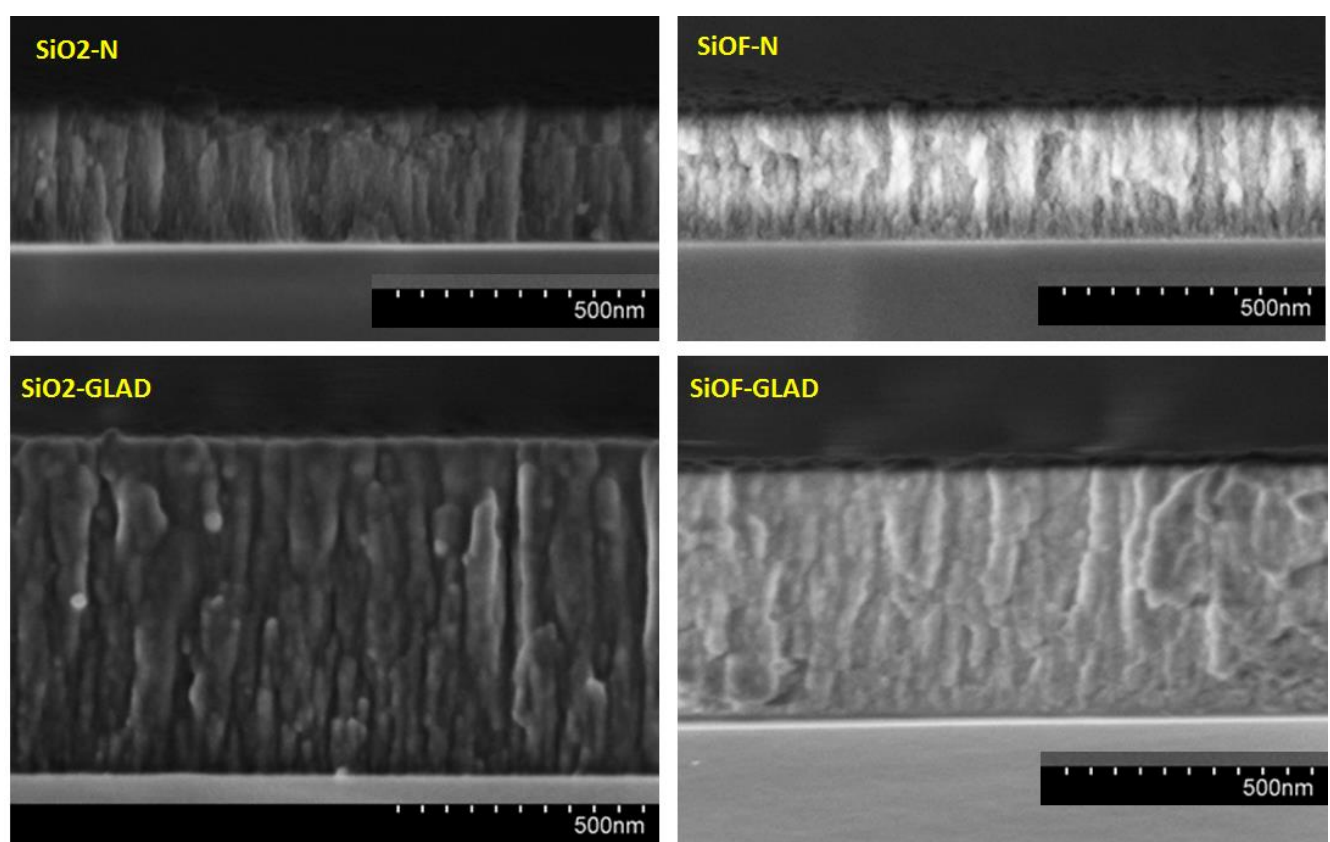

Figure 2. Cross section SEM micrographs of $\mathrm{SiO}_{2}$ and SiOF films. Top: normal geometry without (SiO2-N) and with $F$ (SiOF-N) species in the plasma discharge. Bottom: glancing geometry without (SIO2-GLAD) and with F (SiOF-GLAD) species in the plasma discharge

Figure 3 shows AFM images of the surface topography of the films. As a common feature, the films are rather flat with surface root mean squared (RMS) roughness always below $3 \mathrm{~nm}$ disregarding the preparation conditions. The surface of the films prepared at normal geometry (SiO2-N) are made of aggregates of about 20-40 nm. The average size of these aggregates was smaller when fluorine species were incorporated to the plasma discharge during film growth (SiOF-N). Besides, the surface RMS roughness increases from about $\sim 2 \mathrm{~nm}$ to $\sim 3 \mathrm{~nm}$ with the incorporation of $\mathrm{CF}_{\mathrm{x}}$ precursor in the discharge. On the other hand, in the case of the films prepared in a glancing geometry, similar surface RMS roughness $(\sim 3 \mathrm{~nm})$ was found for films prepared with or without fluorine species in the discharge.
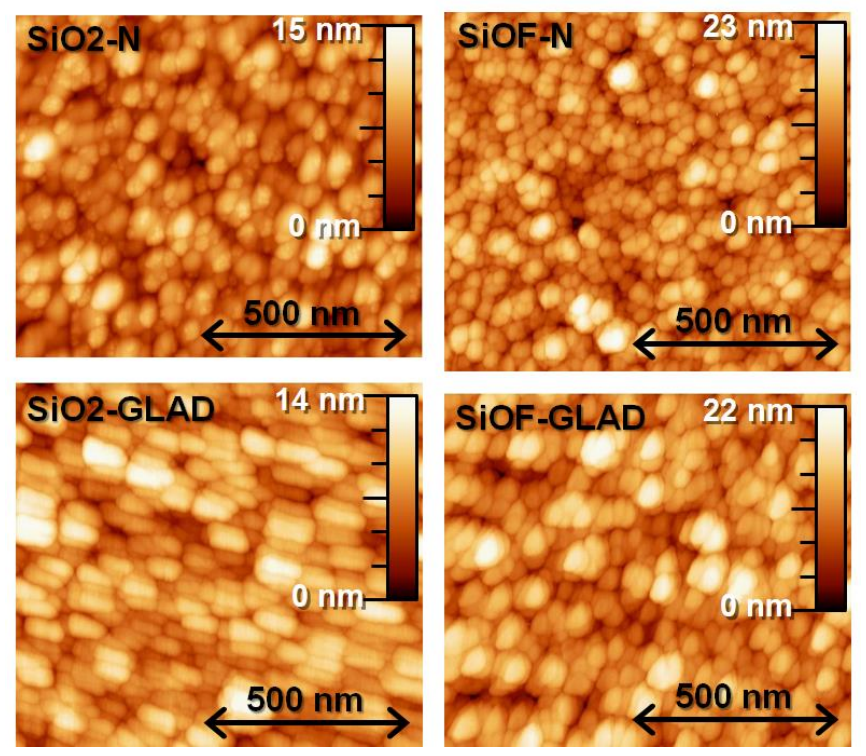

Página 6 de 16 
Figure 3. AFM topographic images of SiOF films prepared in normal (top) and glancing (down) geometry, with (right) or without (left) fluorine species in the plasma discharge

\section{Composition and chemical analysis}

Figure 4 shows a typical survey XPS spectrum of the SiOF films prepared with fluorine species in the plasma discharge. The surface of the samples is mainly composed by silicon and oxygen atoms. Both the $\mathrm{Si} 2 \mathrm{p}$ and $\mathrm{O} 1 \mathrm{~s}$ signals were single peaks (see inset) with a binding energies of $103.3 \mathrm{eV}$ and $532.5 \mathrm{eV}$ respectively, in agreement with a $\mathrm{Si}^{4+}$ oxidation state. Besides, the intensity ratio between the $\mathrm{O} 1 \mathrm{~s}$ and $\mathrm{Si} 2 \mathrm{p}$ peaks was also roughly consistent with a $\mathrm{SiO}_{2}$ stoichiometry. We observe tiny contributions of $\mathrm{C}(\mathrm{C}$ 1s single peak at $284.5 \mathrm{eV}$, reference binding energy) and $\mathrm{F}(\mathrm{F}$ 1s at $686.6 \mathrm{eV})$. The $\mathrm{C}$ content at the sample surface was always below $2-3 \%$ atomic percentage. Silica samples prepared without fluorine species in the plasma discharge presented similar XPS spectra but without the tiny F 1s contribution to the spectra. Note that the XPS characterization was performed for samples as prepared, without applying any surface cleaning process.
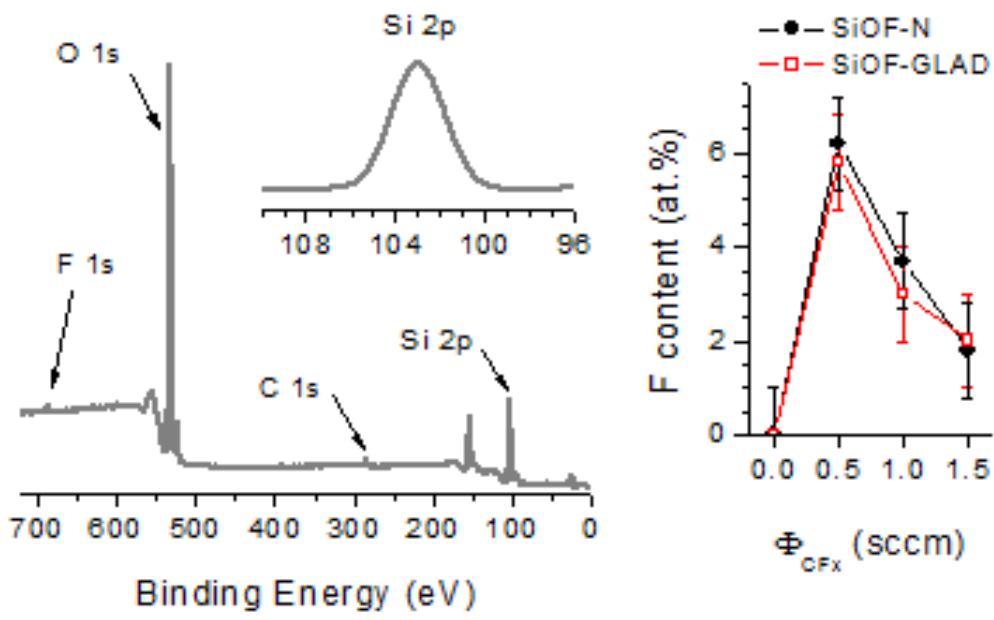

Figure 4. Left: Typical XPS survey spectrum of the porous SiOF films prepared with fluorine species in the plasma discharge as described in this work; inset: blow up of the Si $2 p$ contribution to the spectrum. Right: Fluorine content of the porous SiOF films as a function of the $C_{X}$ mass flow rate in the reaction gas.

The atomic percentage of $\mathrm{F}$ atoms in the whole thickness of the films was determined by PIGE and p-RBS analysis [18-ferrer2012]. It is found that the F content in the films is 6 at.\% when a $\Phi_{\mathrm{CFx}}=0.5 \mathrm{sccm}$ is added to the plasma gas. When the amount of $\mathrm{CF}_{\mathrm{X}}$ increases, the $\mathrm{F}$ incorporation in the film matrix decreases. No significant differences are observed between film samples prepared at normal and glancing deposition geometries.

Figure 5 (left) shows absorption FT-IR spectra of a series of SiOF thin films deposited on intrinsic $\mathrm{Si}(100)$ wafers. The spectra are normalized to have the same 
area. The more intense peak at about $1065-1090 \mathrm{~cm}^{-1}$ has been extensively reported as the Si-O-Si asymmetrical stretching transverse-optical (TO) absorption band $v_{\mathrm{Si}-\mathrm{O}}$. This band shifts to higher energies when changing from normal to glancing geometry or when $\mathrm{CF}_{\mathrm{x}}$ species are incorporated to the plasma discharge. This blue shift is accompanied by a narrowing of the band. Figure 5 (right) shows the evolution of the stretching mode vibration $v_{\mathrm{Si}-\mathrm{O}}$ of $\mathrm{Si}-\mathrm{O}$ bonds as the $\mathrm{CF}_{\mathrm{X}}$ precursor is added to the discharge. A maximum blue shift is achieved for $0.5 \mathrm{sccm}$ of $\mathrm{CF}_{\mathrm{X}}$ incorporated to the plasma discharge for both normal and glancing SiOF films. If the amount of $\mathrm{CF}_{\mathrm{x}}$ in the process gas further increases, a recovery of the peak position to $\sim 1080 \mathrm{~cm}^{-1}$ for both deposition geometries is obtained. Figure 5 also shows a shoulder at $1150 \mathrm{~cm}^{-1}$ due to the longitudinal-optical (LO) absorption mode. Another peak at about $800 \mathrm{~cm}^{-1}$ is due to $\mathrm{Si}-\mathrm{O}$ LO bending absorption. Finally, when $\mathrm{CF}_{\mathrm{x}}$ gas is included to the plasma discharge a new band at about $935 \mathrm{~cm}^{-1}$ appears in the spectrum. This peak has been correlated to either Si-OH [21] or Si-F bond vibrations [12]. Below we will discuss in more detail the origin of these bands.

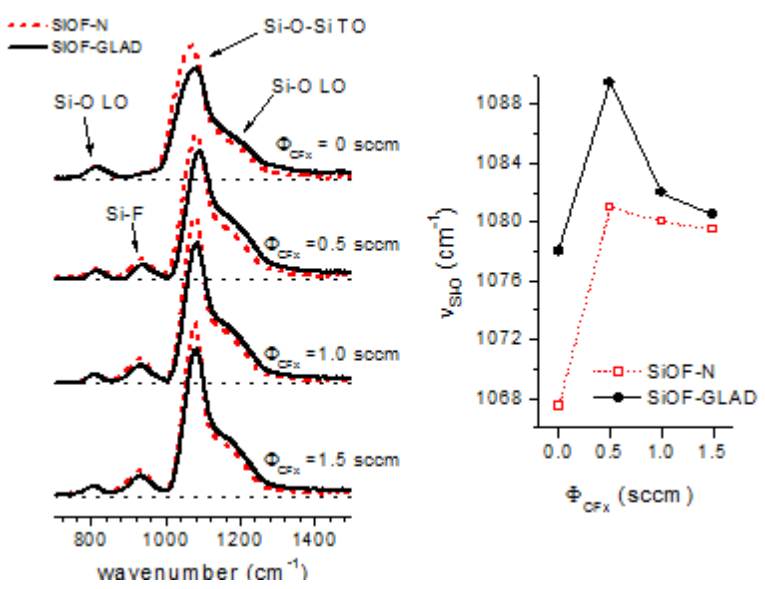

Figure 5. Left: FT-IR spectra of SiOF samples prepared at normal and glancing geometries with several amount of fluorine species in the plasma discharge. Right: Dependence of peak position of the stretching mode vibration $v_{\text {Si-O }}$ on the mass flow rate of $C F_{x}$ precursor in the discharge.

\section{Optical analysis}

Figure 6 shows transmission UV-Vis spectra of porous SiOF films prepared with fluorine species in the plasma discharge at normal (SiOF-N) and glancing (SiOFGLAD) geometries on fused silica plates. The spectrum of the bare fused silica plate is also included in the figure. We observe that the porous SiOF deposits are highly transparent (both in the UV and the visible regions). The interference fringes, denoting a higher transmittance than that of the substrate plate indicates that the refractive index of the deposited films is lower than that of the fused silica substrate $(1.45$ at $550 \mathrm{~nm})$. 


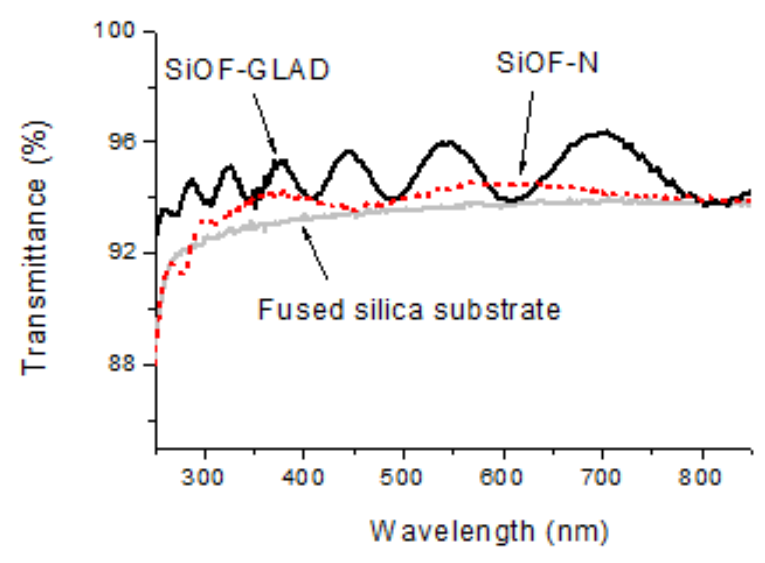

Figure 6. Transmission UV-Vis spectra of SiOF films prepared with $\mathrm{CF}_{x}$ species in the plasma discharge at normal (SiOF-N) and glancing (SiOF-GLAD) geometries.

Quantitative evaluation of the refractive index of the films was done by spectroscopic ellipsometry on samples deposited on $\mathrm{Si}(100)$ wafers. A standard isotropic refractive index model was needed to get the best fits for samples prepared at normal deposition geometry. On the other hand, an uniaxial birefringence model, considering two independent refractive index in-plane, $n_{x}$, and out-of-plane, $n_{z}$, were needed in case of the GLAD films. The results of the analysis are included in Figure 7. This quantification was consistent with the simulation of the interference fringes observed in the UV-Vis transmission experiments.

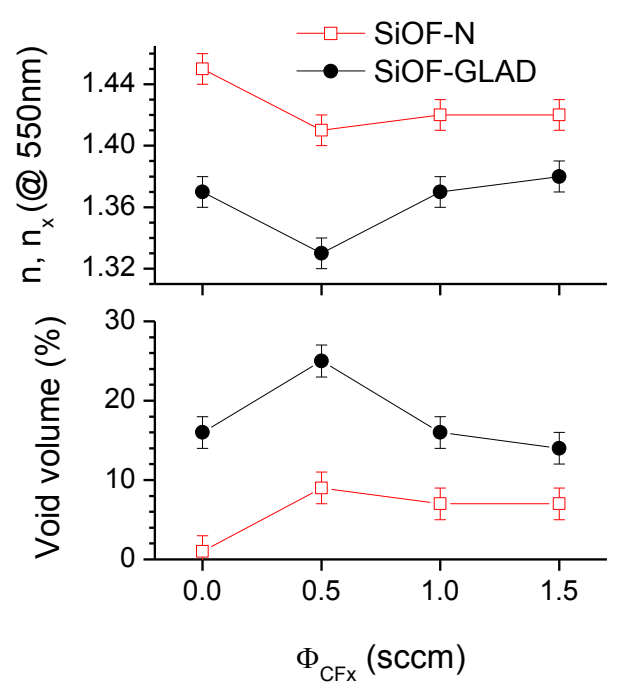

Figure 7. Top: Refractive index (at $550 \mathrm{~nm}$ ) of SiOF films prepared at normal and glancing geometries as a function of the mass flow rates of $\mathrm{CF}_{x}$ in the plasma discharge. Bottom: Void fraction of the films obtained from the optical analysis.

It is found that the refractive index (evaluated at $550 \mathrm{~nm}$ ) of the SiOF films prepared in normal conditions (SiOF-N samples) decreases from 1.45 to 1.41-1.42 when a 
$\Phi_{\mathrm{CFx}}=0.5 \mathrm{sccm}$ is incorporated in the plasma discharge. In these cases, good fitting of the ellipsometric parameters was achieved considering a isotropic film structure. This corresponds to an incorporation of $9-7 \%$ void fraction to the films according to the effective medium description of porous media.

The use of glancing deposition geometry has two effects in the optical response of the films. On the one hand, it induces a lowering of the refractive index of the films. If $\mathrm{CF}_{\mathrm{x}}$ species are not incorporated to the plasma discharge, the refractive index takes values of 1.37-1.38. If $0.5 \mathrm{sccm}$ of $\mathrm{CF}_{\mathrm{X}}$ is introduced in the process gas, it further decreases to $1.33-1.34$. This last value corresponds to a $25 \%$ voids in the films. On the other hand, glancing deposition induces birefringence (i.e., there is an anisotropy in the refractive index). This can be qualitatively observed looking at the magnitude of the non-diagonal elements of the Jones matrix obtained from the spectroscopic ellipsometry measurements (not shown). Thus, it is found that a negative uniaxial birefringence (i.e., $n_{x}<n_{z}$ ) is present at the GLAD SiOF films. The birefringence magnitude $\Delta n\left(\Delta n=n_{x}-n_{z}\right)$ is -0.01 independently of the presence of fluorine species in the plasma discharge during film growth.

\section{DISCUSSION}

It is well known that the use of glancing deposition geometries is a suitable method to increase porosity of thin films. It is extensively reported that the so-called sculptural films can be produced by this experimental approach. This is in fact the case of part of the reported data in this paper. Sculptural silica films are often produced by electron beam evaporation physical vapor deposition (EBE-PVD). They can be highly porous. It is reported that EBE-PVD silica films prepared with deposition angles of $80^{\circ}$ may have refractive index of about 1.25 , which corresponds to $\sim 43 \%$ voids within the films [16]. In our experimental MS configuration for the same deposition angle, we obtain SiO2-GLAD films with $15 \%$ voids (c.f. Figure 7 ). This lower porosity can be understood taking into account that the kinetic energy of the moieties reaching the substrate surface in MS deposition is larger (in the order of few eVs) than that in the case of thermal evaporation (in the range of hundredths of $\mathrm{eV}$ ). Note the short magnetron target to sample distance $(10 \mathrm{~cm})$ used in this work. Besides, a plasma shield develops at the sample surface during film growth due to the presence of the plasma discharge, with the corresponding surface polarization, that in our case is about $20 \mathrm{~V}$. Thus, it is expected a certain amount (few percent of the total gas molecules impinging the surface) of positive ion bombardment $\left(\mathrm{Ar}^{+}, \mathrm{O}_{2}{ }^{+}\right)$during film growth that will contribute to increase the mobility of the deposited silica moieties, and therefore to diminish the corresponding shadowing effects when deposition at glancing angles. It is worth mentioning that the columnar growth observed for MS SiO2-GLAD films prepared under our experimental conditions shows closed pack column, without the clear macroporosity that is observed in EBE-PVD silica films [16]. 
The increase of void fraction of the SiOF films prepared by plasma chemical vapor deposition with fluorinated precursors is usually explained by the incorporation of $\mathrm{Si}$ $\mathrm{F}$ groups within the silica matrix, that precludes the close packing of the $\mathrm{Si}-\mathrm{O}$ chains, increasing the free volume fraction in the films [13]. In fact, we have found that the increase of porosity in SiOF films prepared by reactive MS with $\mathrm{CF}_{\mathrm{x}}$ gas in the plasma discharge correlates to the incorporation of $F$ within the silica network. In both normal and glancing deposition geometries the more porous films contain about a 6 at.\% fluorine atoms, that is achieved including a small amount $(0.5 \mathrm{sccm})$ of $\mathrm{CF}_{\mathrm{x}}$ in the reactive gas during film growth. Increasing further the amount of $\mathrm{CF}_{\mathrm{x}}$ precursor in the plasma discharge has the effect of decreasing the void fraction in the films. Thus, a different mechanism has to be claimed to justify the observed behavior for $\mathrm{CF}_{\mathrm{X}}$ doses above $1 \mathrm{sccm}$ in the discharge.

According to the OES characterization, the fragmentation of the $\mathrm{CF}_{\mathrm{x}}$ gases in the plasma discharge is complete, most probably with the formation of chemically active $\mathrm{F}^{*}$ species. Note that no sign of $\mathrm{C}-\mathrm{F}_{\mathrm{x}}$ species are observed in the plasma discharge, that otherwise are easily observed in other type of less reactive plasmas [22]. This interpretation is also supported by the fact that no significant differences were observed when either $\mathrm{CF}_{4}$ or $\mathrm{C}_{-} \mathrm{C}_{4} \mathrm{~F}_{8}$ were used as fluorine precursors. These, $\mathrm{F}^{*}$ species may act as mild chemical etching agents, that react to $\mathrm{Si}$ moieties surface at the film surface not covalently bonded to the silica matrix to form volatile $\mathrm{SiF}_{\mathrm{x}}$ species that scape from the film surface while the film grows. Note that $\mathrm{CF}_{4}$ and $\mathrm{c}-\mathrm{C}_{4} \mathrm{~F}_{8}$ gases are often used as etching agents $[23,24]$. The etching capabilities of the $F^{*}$ agent increases as the amount of $\mathrm{CF}_{\mathrm{x}}$ in the plasma discharge is increased.

Thus, the porosity in these SiOF films is built by the competition of the incorporation of Si-F groups within the silica matrix, the etching processes induced by the fluorine species and the growth induced by the silicon moieties from the magnetron source and oxygen from the process gas. For low $\mathrm{CF}_{\mathrm{x}}$ doses $\left(\Phi_{\mathrm{CFx}} \sim 0.5 \mathrm{sccm}\right)$ in the plasma discharge, the fluorine species preferentially incorporates to the silica matrix, while for high $\mathrm{CF}_{\mathrm{x}}$ doses $\left(\Phi_{\mathrm{CF} \mathrm{x}} \geq 1.0 \mathrm{sccm}\right)$ etching processes at the sample surface are enhanced through the formation of volatile $\mathrm{SiF}_{\mathrm{x}}$ species before strong covalent bond formation to the silica matrix of the growing film. Note that in this interpretation the etching processes do not contribute to the increase the porosity of the films. Figure 8 shows a schematic representation of the growth mechanisms of porous SiOF thin films described above. 


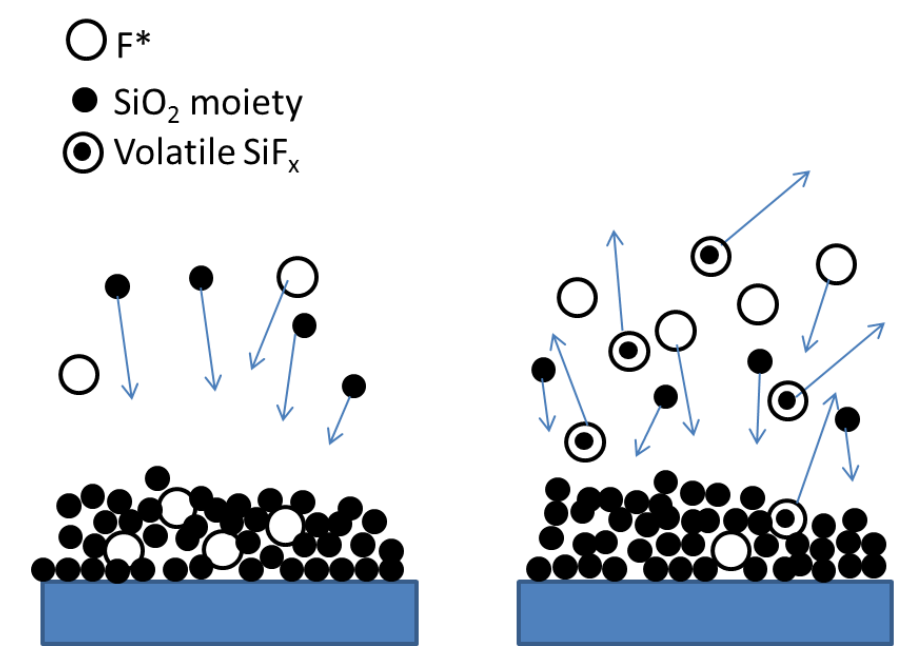

Figure 8. Schematic representation of the growing process of SiOF by reactive magnetron sputtering with mixtures $\mathrm{Ar} / \mathrm{O}_{2} / \mathrm{CF}_{x}$ as plasma gas. Left: low $\mathrm{CF}_{x}$ dose in the process gas. Right: highCF $F_{x}$ dose in the process gas.

Thus, the diminishing of refractive index with the incorporation of $\mathrm{CF}_{\mathrm{x}}$ in the process gas and the corresponding increase of void fraction can be mainly ascribed to the $\mathrm{Si}$ $\mathrm{F}$ bond formation within the film. According to this interpretation, the FTIR band at $925 \mathrm{~cm}^{-1}$ would be due to Si-F bonds and it would be a signature of the porosity of the films. Besides, the transition between dominant $\mathrm{C}-\mathrm{F}$ bond type incorporation to the film network and release of volatile $\mathrm{SiF}_{\mathrm{x}}$ species can be clearly seen in the evolution of the shape and position of the main $\mathrm{Si}-\mathrm{O}-\mathrm{Si}$ stretching band shown in Figures 5 and in the refractive index evolution depicted in Figure 7.

The incorporation of fluorine precursors during standard reactive MS thin film growth can easily be implemented to further decrease the intrinsic low refractive index of silica thin films or to introduce a certain degree of porosity in the films. Thus, for example, multilayer systems of alternative low and high refractive index layers could be developed by pulsing the incorporation of $\mathrm{CF}_{\mathrm{x}}$ precursors in the plasma discharge using a single silicon target.

The combined effect of glancing deposition and Si-F bond formation within the silica matrix during reactive MS SiOF film growth further increases the void content in the films and therefore reduces their refractive index (see Figure 7) especially for low $\mathrm{CF}_{\mathrm{X}}$ doses in the reactive gas.

Besides the general lower refractive index of the SiOF-GLAD with respect to the SiOF-N films, another important difference between them is that the former present negative uniaxial birefringence with a magnitude of about -0.01 (i.e., the light travelling parallel to the surface goes "faster" than in the direction normal to the surface). This might be explained by the higher material connectivity along the columnar structure than in paths within the film across columns. In fact, we may expect easier polarization along the columns than across them. Macroscopic 
polarizability is related to the refractive index through the Clausius-Mossoti relationship. Thus, the macroscopic columnar growth may qualitatively justify the observed negative uniaxial birefringence behavior.

This property may be used to develop thin film based birefringence devices. Note that despite the fact that there is not a columnar structure in the SiOF-GLAD films in the cross section SEM analysis, some degree of uniaxial order is retained as a consequence of the shadowing effects present during the thin film glancing angle deposition. The analysis of the spectroscopic ellipsometry data shows that the incorporation of both $\mathrm{Si}-\mathrm{F}$ bonds and the eventual presence of etching processes when the content of fluorine species in the discharge is enhanced does not modify the magnitude of the uniaxial birefringence of the films.

\section{CONCLUSIONS}

Porous SiOF thin films can be prepared by reactive magnetron sputtering using silicon targets and the incorporation $\mathrm{CF}_{\mathrm{x}}$ species in the plasma discharge during film growth. The porosity is produced by incorporation of $\mathrm{C}-\mathrm{F}$ bonds within the silica network and the competition between film growth due to the sputtered material from the target cathode (and the oxygen process gas) and the surface chemical etching processes induced by the fluorine species. Deposition at glancing angles increases further the void fraction incorporated to the films due to shadowing effects. These porous based silica films are highly transparent in the UV region with refractive indices of 1.41 and 1.33 for normal and glancing deposition geometries (i.e., 9 and $25 \%$ void fraction, respectively). The porosity of the films is correlated to their $F$ content (up to 6 at.\%), as it is often reported for SiOF films [13]. The SiOF films prepared in glancing geometries present negative uniaxial birefringence with a magnitude of -0.01 that is not affected by the presence of $\mathrm{Si}-\mathrm{F}$ bonds within the silica matrix.

\section{Acknowledgements}

Financial support from Junta de Andalucía (Projects P09-CTS- 5189, TEP5283 and FQM-6900), and the Spanish Ministry of Economy and Competitiveness (Projects CONSOLIDER CSD2008-00023, MAT2010-21228, and MAT2010-18447) is acknowledged. 


\section{References:}

[1] K. Maex, M. R. Baklanov, D. Shamiryan and F. lacopi, S. H. Brongersma, Z. S. Yanovitskaya, J. Appl. Phys. 93 (2003) 8793.

[2] S. Chattopadhyay, Y.F. Huang, Y.J. Jen, A. Ganguly, K.H. Chen, L.C. Chen, Mat. Sci. Eng. R 69 (2010) 1.

[3] N.F. Wang, T.W. Kuo, Y.-Z.Tsai, S.-X.Lin, P.-K.Hung, C.-L.Lin, M.-P.Houng, Optics Express 20 (2012) 7445.

[4] M. Oliveros, L. González-García, V. Mugnaini, F. Yubero, N. Roques, J. Veciana, A.R. González-Elipe, C. Rovira, Langmuir 27 (2011) 5098.

[5] J.D. Mackenzie, A.P. Bescher, J. Sol-Gel Sci. Technol. 19 (2000) 23.

[6] A. Barranco, J. Cotrino, F. Yubero, T. Girardeau, S. Camelio, C. Clerc, A.R. González-Elipe, J. Vac. Sci. Technol. A 21 (2003) 900.

[7] L. Martinu, D. Poitras, J. Vac. Sci. Technol. A 18 (2000) 2619.

[8] S.P. Kim, S.K. Choi, Thin Solid Films 379 (2000) 259.

[9] J. Zhang, E.R. Fisher, J. Appl. Phys. 96 (2004) 1094.

[10] M. Cwil, M. Kalisz, P. Konarski, Appl. Surf. Sci. 255 (2008) 1334.

[11] M.D. Barankin, T.S.Williams, E. González II, R.F.Hicks, Thin Solid Films 519 (2010) 1307.

[12] J.C. Alonso, X.M. Díaz-Bucio , A. Ortiz, A. Benami, J.C. Cheang-Wong, L. Rodriguez-Fernández, J. Vac. Sci. Technol. A 25 (2007) 448.

[13] Y-H. Kim, M.S. Hwang, H.J. Kim, J.Y. Kim, Y. Lee, J. Appl. Phys. 90 (2001) 3367.

[14]Y.L. Cheng, Y.L.Wang, H.W.Chen, J.L.Lan, C.P.Liu, S.A.Wu, Y.L.Wu, K.Y. Lo, M.S. Feng, J. Vac. Sci. Tecnol. A 22 (2004) 494.

[15] Z.W.He, D.Y.Xu, X.H.Jiang, Y.Y.Wang, Micropor. Mesopor. Mater. 111 (2008) 206.

[16] L. González-García, G. Lozano, A. Barranco, H. Míguez, A.R. González-Elipe, Mater. Chem. 20 (2010) 6408.

[17] J. Gil-Rostra, M. Cano, J.M. Pedrosa, F.J. Ferrer, F. García-García, F. Yubero, A.R. González-Elipe, ACS Appl. Materials and Interfaces 22 (2012) 628.

[18] F.J. Ferrer, J. Gil-Rostra, A. Terriza, G. Rey, C. Jiménez, J. García-López, F. Yubero, Nuclear Instruments Methods in Physics Research B 274 (2012) 65. 
[19] A. Walkiewicz-Pietrzkowska, J. Cotrino, A.R. González-Elipe, Chem. Vap. Deposition 11 (2005) 317.

[20] R. W. B. Pearse, A. G. Gaydon, The Identification of Molecular Spectra, 4th edition, Chapman and Hall, London 1976

[21] A. Barranco, J. Cotrino, F. Yubero, T. Girardeau, S. Camelio, A.R. GonzálezElipe, Surf. Coat. Technol. 181 (2004) 244.

[22] R. d' Agostino, F. Cramarossa, F. Illuzzi, J. Appl. Phys. 61 (1987) 2754.

[23] K. Nojiri, E. Iguchi J. Vac. Sci. Technol. B 13 (1995) 1451.

[24] H. Abe, M. Yoneda, N. Fujiwara, Jpn. J. Appl. Phys. 47 (2008) 1435. 


\section{Figure Captions:}

Figure 1. OES spectra measured at the plasma discharge during SiOF film growth by reactive magnetron sputtering.

Figure 2. Cross section SEM micrographs of $\mathrm{SiO}_{2}$ and SiOF films. Top: normal geometry without (SiO2-N) and with $\mathrm{F}(\mathrm{SiOF}-\mathrm{N})$ species in the plasma discharge. Bottom: glancing geometry without (SiO2-GLAD) and with F (SiOF-GLAD) species in the plasma discharge

Figure 3. AFM topographic images of $\mathrm{SiO}_{2}$ and $\mathrm{SiOF}$ films prepared in normal (top) and glancing (down) geometry, with (right) or without (left) fluorine species in the plasma discharge

Figure 4. Left: Typical XPS survey spectrum of the porous SiOF films prepared with fluorine species in the plasma discharge as described in this work; inset: blow up of the Si $2 p$ contribution to the spectrum. Right: Fluorine content of the porous SiOF films as a function of the $\mathrm{CF}_{\mathrm{x}}$ mass flow rate in the reaction gas.

Figure 5. Left: FT-IR spectra of SiOF samples prepared at normal and glancing geometries with several amount of fluorine species in the plasma discharge. Right: Dependence of peak position of the stretching mode vibration $v_{\mathrm{Si}-\mathrm{O}}$ on the mass flow rate of $\mathrm{CF}_{\mathrm{x}}$ precursor in the discharge.

Figure 6. Transmission UV-Vis spectra of SiOF films prepared with $\mathrm{CF}_{\mathrm{x}}$ species in the plasma discharge at normal (SiOF-N) and glancing (SiOF-GLAD) geometries.

Figure 7. Top: Refractive index (at $550 \mathrm{~nm}$ ) of SiOF films prepared at normal and glancing geometries as a function of the mass flow rates of $\mathrm{CF}_{\mathrm{X}}$ in the plasma discharge. Bottom: Void fraction of the films obtained from the optical analysis.

Figure 8. Schematic representation of the growing process of SiOF by reactive magnetron sputtering with mixtures $\mathrm{Ar} / \mathrm{O} 2 / \mathrm{CF}_{\mathrm{x}}$ as plasma gas. Left: low $\mathrm{CF}_{\mathrm{x}}$ dose in the process gas. Right: high CFx dose in the process gas. 